\title{
BOOSTER STOPBAND CORRECTIONS
}

\section{BOOSTER TECHNICAL NOTE}

NO. 217

\author{
C. J. GARDNER
}

January 6, 1993 


\title{
BOOSTER STOPBAND CORRECTIONS
}

\author{
C. J. Gardner
}

January 6, 1993

\section{HALF-INTEGER RESONANCE CORRECTIONS}

\subsection{Purpose}

The purpose of these corrections is to eliminate those components of quadrupole fields in the booster which excite the $2 Q_{x}=9$ and $2 Q_{y}=9$ resonances. These resonances must be corrected simultaneously without altering the machine tunes or introducing any unwanted harmonics.

\subsection{Magnets Used}

Each superperiod of the booster contains eight quadrupoles which are labled QVX1, QHX2, QVX3, QHX4, QVX5, QHX6, QVX7, and QHX8, where $Q H$ and $Q V$ denote horizontal and vertical focusing quadrupoles, and $\mathrm{X}$ refers to superperiod $\mathrm{A}, \mathrm{B}, \mathrm{C}, \mathrm{D}, \mathrm{E}$, or $\mathrm{F}$. These are the main quadrupoles of the booster lattice. Each quadrupole has a main winding consisting of 5 turns and an auxiliary winding (or trim winding) consisting of 2 turns. The measured integrated strengths of the QH and QV quadrupoles (see Refs. 4 and 5) are respectively 1.834 and 1.877 gauss per ampere-turn. The auxiliary windings are used for the half-integer resonance correction and are refered to here by the names of the quadrupoles on which they are wound.

The auxiliary windings on the quads are connected together to form four strings which are labeled QVSTR1, QHSTR1, QVSTR2, and QHSTR2. Each string consists of the auxiliary windings of 12 of the quads connected 
in series as indicated below. The 't' and '-' signs indicate the polarity of each auxiliary winding in the string.

QVSTR1: +QVA1 +QVA7 -QVB1 -QVB7 +QVC1 +QVC7 -QVD1

-QVD7 +QVE1 +QVE7 -QVF1 -QVF7.

QHSTR1: +QHA2 +QHA8-QHB2-QHB8 +QHC2 +QHC8 -QHD2

-QHD8 +QHE2 +QHE8 -QHF2 -QHF8.

QVSTR2: +QVA3 -QVA5 -QVB3 +QVB5 +QVC3 -QVC5 -QVD3

+QVD5 +QVE3 -QVE5 -QVF3 +QVF5.

QHSTR2: +QHA4-QHA6 -QHB4 +QHB6 +QHC4 -QHC6 -QHD4

+QHD6 +QHE4 -QHE6 -QHF4 +QHF6.

The division of the 48 auxiliary windings into four strings with the indicated magnets and polarities insures that no $10 \theta, 5 \theta, 4 \theta$, or $0 \theta$ harmonic components are produced by any of the quadrupole strings. The details of this scheme are discussed in Refs. (1-2) and in the appendix.

The 12 auxiliary windings in each string are connected together with number 2 (AWG) cable. The total resistance of each series string, including the resistance of the windings, is $0.624 \mathrm{ohms}$. The estimated inductance of each string is $0.77 \mathrm{mH}$. The net EMF induced in each string by the currents in the main windings of the magnets is zero.

\subsection{Excitation Scheme}

The four strings QVSTR1, QHSTR1, QVSTR2, QHSTR2 are excited with currents $J_{1}, J_{2}, J_{3}$, and $J_{4}$, respectively. In Refs. (1) and (2) it is shown that the $2 Q_{x}=9$ and $2 Q_{y}=9$ resonance excitation components $C X, S X$, $C Y$, and $S Y$ produced by these currents are given by

$$
\left(\begin{array}{c}
C X \\
S X \\
C Y \\
S Y
\end{array}\right)=N\left(\frac{e}{c P}\right) \mathrm{MQ}\left(\begin{array}{c}
J_{1} \\
J_{2} \\
J_{3} \\
J_{4}
\end{array}\right)
$$

where

$$
\mathbf{M}=\left(\begin{array}{rrrr}
28.139 & -76.062 & -37.769 & -126.518 \\
36.050 & 127.317 & 27.678 & -77.381 \\
124.432 & -9.590 & -85.089 & -45.423 \\
85.027 & 45.418 & 124.537 & -9.564
\end{array}\right)
$$




$$
\mathbf{Q}=\left(\begin{array}{cccc}
Q_{V} & 0 & 0 & 0 \\
0 & Q_{H} & 0 & 0 \\
0 & 0 & Q_{V} & 0 \\
0 & 0 & 0 & Q_{H}
\end{array}\right)
$$

$Q_{H}=3.668 \times 10^{-4}$ and $Q_{V}=3.754 \times 10^{-4}$ (tesla per ampere) are the integrated quadrupole strengths produced by each 2-turn auxiliary winding on the $\mathrm{QH}$ and $\mathrm{QV}$ quadrupoles, $P$ is the momentum, $c P / e=3.335641$ tesla-m per $\mathrm{GeV}$, and the normalization constant, $N=10^{5} /(2 \pi)$, is such that $\sqrt{C X^{2}+S X^{2}}$ and $\sqrt{C Y^{2}+S Y^{2}}$ are $10^{5}$ times the resonance stopband widths produced by the correction scheme. The matrix elements in (2) are those obtained by Tepikian in Ref. (2) and are expressed in meters.

The currents required to produce a given set of excitation components are given by

$$
\left(\begin{array}{c}
J_{1} \\
J_{2} \\
J_{3} \\
J_{4}
\end{array}\right)=\frac{1}{N}\left(\frac{c P}{e}\right) \mathbf{Q}^{-1} \mathbf{M}^{-1}\left(\begin{array}{c}
C X \\
S X \\
C Y \\
S Y
\end{array}\right)
$$

where

$$
\mathbf{M}^{-1}=\frac{1}{1000}\left(\begin{array}{rrrr}
-1.365 & -1.833 & 6.054 & 4.130 \\
-3.916 & 6.347 & 0.560 & -2.215 \\
1.868 & -1.357 & -4.165 & 6.052 \\
-6.411 & -3.819 & 2.253 & 0.444
\end{array}\right)
$$

Thus if the operator selects values for $C X, S X, C Y$, and $S Y$ then substitution of these values into equation (4) will give the currents required in the four strings. Alternatively, if the operator wants to know the values of the excitation components produced by the correction scheme for a given set of currents, then these values are obtained by substituting the currents into equation (1).

\subsection{Power Supplies}

Each of the four strings of auxiliary windings is connected to its own power supply. These power supplies are bipolar and can deliver a maximum current of 12.5 amperes at a maximum of 50 volts. Each 2-turn auxiliary winding therefore provides a maximum excitation of 25 ampere-turns. This 
is $0.22 \%$ of the maximum excitation of 11500 ampere-turns provided by each 5-turn main winding during proton extraction. The maximum current requirement is based in part on Tepikian's calculation [3] of the quadrupole strength required to correct the resonances. Since the resistance of each string is 0.624 ohms, the minimum voltage required at 12.5 amperes is 8 volts. The additional voltage is sufficient to ramp the current in each string from zero to the maximum current in half the booster cycle period of 0.133 seconds.

\subsection{Controls}

The current in each string of quadrupoles is programed with a 400 point function generator which has the same name as the quadrupole string. The 400 points are assigned values determined by the desired program and are clocked at a rate of one point per ms for proton acceleration, and at a rate of one point every two ms for heavy ion acceleration. The first point of the function generator is clocked out at $T_{0}$ and the function is held at this value until the function generator recieves a start pulse at time $T_{\text {start }}$ which is specified by the user. When the start pulse is recieved, the function generator points are clocked out at the specified rate of one or two points per ms. This continues until all 400 points have been clocked out, or until the function generator recieves a stop pulse. The time, $\mathrm{T}_{\text {stop }}$, of the stop pulse is specified by the user and must be at least $25 \mathrm{~ms}$ before $T_{0}$ for the next machine cycle. When the function generator recieves a stop pulse the clocking of points stops and the function remains at the value it had at time $T_{\text {stop. }}$. If all 400 points are clocked before a stop pulse occurs then the function remains at the value of the 400th point.

The function for each of the four strings is normally obtained from the functions specified by the user for the four excitation components. The currents required in the four strings are then obtained from equation (4) with $P=0.645 \mathrm{GeV} / \mathrm{c}$. The user may sometimes find it useful to directly specify the functions for the four strings. The values of the excitation components are then obtained from equation (1) with $P=0.645 \mathrm{GeV} / \mathbf{c}$. The value of the current in each of the four strings may be read back at time $\mathrm{T}_{\text {sample }}$ which is specified by the user. Analog signals for each string are available through a multiplexer. 


\section{THIRD-INTEGER RESONANCE CORRECTIONS}

\subsection{Purpose}

The purpose of these corrections is to eliminate those components of sextupole fields in the booster which excite the $3 Q_{x}=14, Q_{x}+2 Q_{y}=14$, $3 Q_{x}=13$, and $Q_{x}+2 Q_{y}=13$ resonances. These resonances must be corrected simultaneously without altering the machine chromaticities or introducing any unwanted harmonics.

\subsection{Magnets Used}

Each superperiod of the booster contains eight sextupoles which are labled SVX1, SHX2, SVX3, SHX4, SVX5, SHX6, SVX7, and SHX8, where SH and SV denote respectively sextupoles located near horizontal and vertical beta maximums, and $\mathrm{X}$ refers to superperiod $\mathrm{A}, \mathrm{B}, \mathrm{C}, \mathrm{D}, \mathrm{E}$, or $\mathrm{F}$. These are the main sextupoles of the booster lattice used to adjust the machine chromaticity. The centers of the sextupoles are located $55.2 \mathrm{~cm}$ upstream of the centers of the quadrupoles-i.e. the center of SVX1 is $55.2 \mathrm{~cm}$ upstream of the center of QVX1, and so on. Each sextupole has a main winding consisting of 8 turns and an auxiliary winding consisting of either one or two turns. The measured integrated strength of each sextupole (see Ref. 6) is $8.215 \times 10^{-4}$ tesla/m per ampere turn. The auxiliary windings are used for the third-integer resonance correction and are refered to here by the names of the sextupoles on which they are wound.

The auxiliary windings on the sextupoles are connected togther to form eight strings which are labeled SVSTR1, SHSTR1, SVSTR2, SHSTR2, SVSTR3, SHSTR3, SVSTR4, SHSTR4. Fach string consists of the auxiliary windings of 6 of the sextupoles connected in series as indicated below. The ' + ' and '-' signs indicate the polarity of each winding in the string, and ' $/ 2$ ' indicates auxiliary windings with just one turn.

SVSTR1: +SVA1 -SVA3 -SVC1/2 +SVC3/2 -SVE1/2 +SVE3/2

SHSTR1: +SHA2 -SHA4 -SHC2/2 +SHC4/2 -SHE2/2 +SHE4/2

SVSTR2: +SVA5 -SVA7 -SVC5/2 +SVC7/2 -SVE5/2 +SVE7/2

SHSTR2: + SHA6 -SHA8 -SHC6/2 + SHC8/2 -SHE6/2 +SHE8/2 
SVSTR3: +SVD1 -SVD3 -SVF1/2 +SVF3/2-SVB1/2+SVB3/2

SHSTR3: +SHD2 -SHD4 -SHF2/2 +SHF4/2-SHB2/2+SHB4/2

SVSTR4: +SVD5 -SVD7 -SVF5/2+SVF7/2-SVB5/2+SVB7/2

SHSTR4: +SHD6 -SHD8 -SHF6/2 +SHF8/2-SHB6/2 +SHB8/2

The division of the 48 auxiliary windings into eight strings with the indicated magnets and polarities insures that no $14 \theta, 10 \theta, 9 \theta, 4 \theta$ or $0 \theta$ harmonic components are produced during correction of the $3 Q_{x}=13$ and $Q_{x}+2 Q_{y}=13$ resonances, and no $13 \theta, 9 \theta, 5 \theta$, or $0 \theta$ harmonic components are produced during correction of the $3 Q_{x}=14$ and $Q_{x}+2 Q_{y}=14$ resonances. The details of this scheme are discussed in Refs. (1-2) and in the appendix.

The 6 auxiliary windings in each string are connected together with number 2 (AWG) cable. The total resistance of each series string, including the resistance of the windings, is $0.3066 \mathrm{ohms}$. The estimated inductance of each string is $0.003 \mathrm{mH}$. The net EMF induced in each string by the currents in the main windings of the magnets is zero.

\subsection{Excitation Scheme}

The eight strings SVSTR1, SHSTR1,..., SHSTR4 are excited with currents $J_{1}, J_{2}, \ldots, J_{8}$ respectively. If $J_{j}=J_{j+4}$ for $j=1,2,3,4$, then the $3 Q_{x}=p$ and $Q_{x}+2 Q_{y}=p$ resonance excitation components for $p=13$ are zero. Similarly, if $J_{j+4}=-J_{j}$ then the resonance excitation components for $p=14$ are zero. Thus, the $3 Q_{x}=14$ and $Q_{x}+2 Q_{y}=14$ resonance excitation components $C X_{14}, S X_{14}, C Y_{14}$, and $S Y_{14}$ produced by the currents in the eight strings are given by

$$
\left(\begin{array}{l}
C X_{14} \\
S X_{14} \\
C Y_{14} \\
S Y_{14}
\end{array}\right)=\frac{N}{2}\left(\frac{e S}{c P}\right) \mathrm{M}_{14}\left(\begin{array}{l}
J_{1}+J_{5} \\
J_{2}+J_{6} \\
J_{3}+J_{7} \\
J_{4}+J_{8}
\end{array}\right)
$$

and the $3 Q_{x}=13$ and $Q_{x}+2 Q_{y}=13$ resonance excitation components $C X_{13}, S X_{13}, C Y_{13}$, and $S Y_{13}$ are given by

$$
\left(\begin{array}{c}
C X_{13} \\
S X_{13} \\
C Y_{13} \\
S Y_{13}
\end{array}\right)=\frac{N}{2}\left(\frac{e S}{c P}\right) \mathbf{M}_{13}\left(\begin{array}{c}
J_{1}-J_{5} \\
J_{2}-J_{6} \\
J_{3}-J_{7} \\
J_{4}-J_{8}
\end{array}\right)
$$


where

$$
\mathbf{M}_{14}=\left(\begin{array}{rrrr}
39.017 & -230.433 & -8.606 & -191.932 \\
34.454 & 60.248 & 56.746 & -147.730 \\
125.164 & -59.570 & 0.214 & -88.629 \\
75.279 & 66.529 & 150.993 & -14.219
\end{array}\right)
$$

and

$$
\mathbf{M}_{13}=\left(\begin{array}{rrrr}
52.718 & -211.124 & 35.220 & -252.319 \\
33.732 & 140.525 & 59.665 & 54.026 \\
148.590 & -46.760 & 103.567 & -83.961 \\
62.797 & 92.117 & 131.038 & 61.137
\end{array}\right) \text {. }
$$

The matricies $\mathbf{M}_{13}$ and $\mathbf{M}_{14}$ are those obtained by Tepikian in Ref. (2). In equations (6) and (7), $S=16.43 \times 10^{-4}$ tesla/m per ampere is the integrated sextupole strength produced by each 2-turn auxiliary winding, $P$ is the momentum, $c P / e=3.335641$ tesla-m per $\mathrm{GeV}$, and the normalization constant, $N=250$. The matrix elements in (8) and (9) are given in units of $m^{3 / 2}$.

The currents required to produce a given set of excitation components are given by

$$
\left(\begin{array}{c}
J_{1} \\
J_{2} \\
J_{3} \\
J_{4}
\end{array}\right)=\frac{1}{N}\left(\frac{c P}{e S}\right)\left[\mathrm{M}_{14}^{-1}\left(\begin{array}{c}
C X_{14} \\
S X_{14} \\
C Y_{14} \\
S Y_{14}
\end{array}\right)+\mathbf{M}_{13}^{-1}\left(\begin{array}{c}
C X_{13} \\
S X_{13} \\
C Y_{13} \\
S Y_{13}
\end{array}\right)\right]
$$

and

$$
\left(\begin{array}{c}
J_{5} \\
J_{6} \\
J_{7} \\
J_{8}
\end{array}\right)=\frac{1}{N}\left(\frac{c P}{e S}\right)\left[\mathbf{M}_{14}^{-1}\left(\begin{array}{c}
C X_{14} \\
S X_{14} \\
C Y_{14} \\
S Y_{14}
\end{array}\right)-\mathbf{M}_{13}^{-1}\left(\begin{array}{c}
C X_{13} \\
S X_{13} \\
C Y_{13} \\
S Y_{13}
\end{array}\right)\right]
$$

where

$$
\mathbf{M}_{14}^{-1}=\frac{1}{1000}\left(\begin{array}{rrrr}
-2.684 & -1.983 & 9.023 & 0.579 \\
-3.987 & 4.681 & 1.150 & -1.988 \\
2.991 & -1.635 & -4.942 & 7.415 \\
-1.103 & -5.950 & 0.675 & 2.172
\end{array}\right)
$$

and

$$
\mathbf{M}_{13}^{-1}=\frac{1}{1000}\left(\begin{array}{rrrr}
-5.969 & 0.561 & 12.238 & -8.323 \\
1.971 & 13.164 & -1.397 & -5.419 \\
4.390 & -4.167 & -6.216 & 13.262 \\
-6.246 & -11.479 & 2.858 & 4.647
\end{array}\right) \text {. }
$$


Thus if the operator selects values for the eight excitation components $C X_{14}, S X_{14}, C Y_{14}, S Y_{14}, C X_{13}, S X_{13}, C Y_{13}$, and $S Y_{13}$, then substitution of these values into equations (10-11) will give the currents required in the eight strings. Alternatively, if the operator wants to know the values of the excitation components produced by the correction scheme for a given set of currents, then these values are obtained by substituting the currents into equations (6-7).

\subsection{Power Supplies}

Each of the eight strings of auxiliary windings is connected to its own power supply. These power supplies are bipolar and can deliver a maximum current of 50 amperes at a maximum of 25 volts. Each 2-turn auxiliary winding therefore provides a maximum excitation of 100 ampere-turns. This is $3.6 \%$ of the maximum excitation of 2800 ampere-turns provided by each 8-turn main winding. The maximum current requirement is based in part on Tepikian's calculation [3] of the sextupole strength required to correct the resonances. Since the resistance of each string is $0.3066 \mathrm{ohms}$, the mimimum voltage required at 50 amperes is 15.33 volts. The additional voltage is sufficient to ramp the current in each string from zero to the maximum current in half the booster cycle period of 0.133 seconds.

\subsection{Controls}

The current in each string of sextupoles is programed with a 400 point function generator (described in section 1.5) which has the same name as the sextupole string. The function for each of the eight strings is normally obtained from the functions specified by the user for the eight excitation components. The currents required in the eight strings are then obtained from equations $(10-13)$ with $P=0.645 \mathrm{GeV} / \mathrm{c}$. The user may sometimes find it useful to directly specify the functions for the eight strings. The values of the excitation components are then obtained from equations (6-9) with $P=0.645 \mathrm{GeV} / \mathrm{c}$.

The value of the current in each of the four strings may be read back at time $\mathrm{T}_{\text {sample }}$ which is specified by the user. Analog signals for each string are available through a multiplexer. 


\section{LINEAR COUPLING RESONANCE CORRECTIONS}

\subsection{Purpose}

The purpose of these corrections is to eliminate those components of skew quadrupole fields in the booster which excite the $Q_{x}-Q_{y}=0$ and $Q_{x}+Q_{y}=9$ resonances. These resonances must be corrected simultaneously without introducing any unwanted harmonics.

\subsection{Magnets Used}

With the exception of superperiods C, D, and F, each superperiod of the booster contains eight correction packages centered $67.1 \mathrm{~cm}$ upstream of the center of each quadrupole. Each package contains a horizontal and a vertical dipole, and an additional set of coils which can be excited to produce either a quadrupole or a skew quadrupole. The packages upstream of quadrupoles QVX1, QHX2, QVX7, and QHX8 contain skew quadrupoles which are labeled QSX1, QSX2, QSX7, and QSX8. These are the skew quadrupoles used for the correction of the $Q_{x}-Q_{y}=0$ and $Q_{x}+Q_{y}=9$ resonances. Each skew quad has a 48 -turn winding and a measured integrated strength (see Ref. 7) of 5.45 gauss per ampere. In superperiod $\mathrm{C}$ there is no correction package upstream of quadrupole QVC5 and in superperiods D and F there is no correction package upstream of quadrupoles QHD6 and QHF6.

The skew quads are connected together to form four strings which are labeled QSSTR1, QSSTR2, QSSTR3, and QSSTR4. Each string consists of six of the skew quads connected in series as indicated below. The polarities of the skew quads in each string are all the same.

QSSTR1: QSA1 QSA7 QSC1 QSC7 QSE1 QSE7

QSSTR2: QSA2 QSA8 QSC2 QSC8 QSE2 QSE8

QSSTR3: QSB1 QSB7 QSD1 QSD7 QSF1 QSF7

QSSTR4: QSB2 QSB8 QSD2 QSD8 QSF2 QSF8

The divison of the 24 skew quads into these four strings allows one to independently correct the $Q_{x}-Q_{y}=0$ and $Q_{x}+Q_{y}=9$ resonances. The details of this scheme are discussed in Ref. (2) and in the appendix. 
The six skew quads in each string are connected together with number 2 (AWG) cable. The total resistance of each series string, including the resistance of the skew quads, is $0.771 \mathrm{ohms}$. The estimated inductance of each string is $12 \mathrm{mH}$.

\subsection{Excitation Scheme}

The four strings QSSTR1, QSSTR2, QSSTR3, and QSSTR4 are excited with currents $J_{1}, J_{2}, J_{3}$, and $J_{4}$ respectively. If $I_{j}=I_{j+2}$ for $j=1,2$, then the $Q_{x}+Q_{y}=9$ resonance excitation components are zero. Similarly, if $J_{j+2}=-J_{j}$ then the $Q_{x}-Q_{y}=0$ resonance excitation components are zero. Thus, the $Q_{x}-Q_{y}=0$ and $Q_{x}+Q_{y}=9$ resonance excitation components produced by the currents in the four strings are given by

$$
\left(\begin{array}{c}
C_{0} \\
S_{0}
\end{array}\right)=\frac{N}{2}\left(\frac{e Q}{c P}\right) \mathbf{N}_{1}\left(\begin{array}{c}
J_{1}+J_{3} \\
J_{2}+J_{4}
\end{array}\right)
$$

and

$$
\left(\begin{array}{c}
C_{9} \\
S_{9}
\end{array}\right)=\frac{N}{2}\left(\frac{e Q}{c P}\right) \mathbf{N}_{2}\left(\begin{array}{c}
J_{1}-J_{3} \\
J_{2}-J_{4}
\end{array}\right)
$$

where

$$
\mathbf{N}_{1}=\left(\begin{array}{rr}
90.254 & 87.046 \\
4.517 & 23.301
\end{array}\right), \quad \mathbf{N}_{2}=\left(\begin{array}{rr}
71.556 & -14.017 \\
43.519 & 82.487
\end{array}\right)
$$

The matricies $\mathbf{N}_{1}$ and $\mathbf{N}_{2}$ are those obtained by Tepikian in Ref. (2). In equations (14) and (15), $Q=5.45 \times 10^{-4}$ tesla per ampere is the integrated strength of each skew quad, $P$ is the momentum, $c P / e=3.335641$ tesla-m per $\mathrm{GeV}$, and the normalization constant, $N=10^{5} / 4 \pi$. The matrix elements in (16) are given in meters.

The currents required to produce a given set of excitation components are given by

$$
\left(\begin{array}{c}
J_{1} \\
J_{2}
\end{array}\right)=\frac{1}{N}\left(\frac{c P}{e Q}\right)\left[\mathbf{N}_{1}^{-1}\left(\begin{array}{c}
C_{0} \\
S_{0}
\end{array}\right)+\mathbf{N}_{2}^{-1}\left(\begin{array}{c}
C_{9} \\
S_{9}
\end{array}\right)\right]
$$

and

$$
\left(\begin{array}{l}
J_{3} \\
J_{4}
\end{array}\right)=\frac{1}{N}\left(\frac{c P}{e Q}\right)\left[\mathbf{N}_{1}^{-1}\left(\begin{array}{c}
C_{0} \\
S_{0}
\end{array}\right)-\mathbf{N}_{2}^{-1}\left(\begin{array}{c}
C_{9} \\
S_{9}
\end{array}\right)\right]
$$


where

$$
\mathbf{N}_{1}^{-1}=\frac{1}{1000}\left(\begin{array}{rr}
13.628 & -50.912 \\
-2.642 & 52.788
\end{array}\right), \quad \mathbf{N}_{2}^{-1}=\frac{1}{1000}\left(\begin{array}{rr}
12.666 & 2.152 \\
-6.682 & 10.988
\end{array}\right)
$$

Thus if the operator selects values for $C_{0}, C_{9}$, and $S_{9}$ then substitution of these values into equations (17-18) will give the currents required in the four strings. Alternatively, if the operator wants to know the values of the excitation components produced by the correction scheme for a given set of currents, then these values are obtained by substituting the currents into equations (14-15).

\subsection{Power Supplies}

Each of the four strings of skew quadrupoles is connected to its own power supply. These power supplies are bipolar and can deliver a maximum current of 25 amperes at a maximum of 32 volts. Each skew quad therefore has a maximum integrated strength of 135 gauss. This is $0.64 \%$ of the maximum integrated strength of 2.1 tesla provided by each main quadrupole during proton extraction. The maximum current requirement is based in part on Tepikian's calculation [3] of the skew quadrupole strength required to correct the resonances. Since the resistance of each string is $0.771 \mathrm{ohms}$, the minimum voltage required at 25 amperes is 19 volts. The additional voltage is sufficient to ramp the current in each string from zero to the maximum current in half the booster cycle period of 0.133 seconds.

\subsection{Controls}

The current in each string of skew quadrupoles is programed with a 400 point function generator (described in section 1.5) which has the same name as the skew quadrupole string. The function for each of the four strings is normally obtained from the functions specified by the user for the four excitation components. The currents required in the four strings are then obtained from equations (17-19) with $P=0.645 \mathrm{GeV} / \mathrm{c}$. The user may sometimes find it useful to directly specify the functions for the four strings. The values of the excitation components are then obtained from equations (14-16) with $P=0.645 \mathrm{GeV} / \mathrm{c}$. 
The value of the current in each of the four strings may be read back at time $T_{\text {sample }}$ which is specified by the user. Analog signals for each string are available through a multiplexer.

\section{Appendix: Sum and Difference Resonances}

A detailed discussion of the theory of sum and difference resonances may be found in Ref.(8). Following are some basic results.

The resonance is defined by the equation

$$
m Q_{x}+n Q_{y}=N
$$

where $Q_{x}$ and $Q_{y}$ are the horizontal and vertical tunes, and $m, n$, and $N$ are integers. If $m$ and $n$ have opposite signs, the resonance is called a difference resonance; otherwise it is called a sum resonance. The order, $l$, of the resonance is

$$
l=|m|+|n| .
$$

If the tunes are sufficiently close to the resonance, i.e. if they are within the resonance stopband, and if $n$ is even (odd), the resonance will be excited by the $N$ th harmonic, in azimuth $\theta$, of the normal (skew) $2 l$-pole fields present in the machine. The resonance condition (20) arises from the first-order perturbation treatment of the vector potential terms $x^{|m|} y^{|n|}$ associated with the $2 l$-pole field. The width of the stopband is proportional to the strength of the $2 l$-pole field, and for resonances of order 3 and higher also depends on the amplitudes, $J_{x}$ and $J_{y}$, of the betatron oscillations. If the tunes are near the resonance, and if $m \neq 0, n \neq 0$, then the quantity

$$
C=n J_{x}-m J_{y}
$$

is a constant of the motion. For the case of the difference resonances this implies that the amplitudes are bounded; for sum resonances the amplitudes can increase without bound. In the presence of space-charge forces the amplitudes are generally bounded as discussed in Ref. (9).

\subsection{Excitation Coefficients}

The excitation coefficient for the resonance (20) is of the form

$$
\kappa=\frac{C}{p} \int_{0}^{2 \pi r} \beta_{x}^{|m / 2|} \beta_{y}^{|n / 2|} K(s) e^{i \psi} d s
$$


where $p$ is the momentum,

$$
\begin{gathered}
\psi(s)=m\left(\mu_{x}-Q_{x} \theta\right)+n\left(\mu_{y}-Q_{y} \theta\right)+N \theta \\
\mu_{x}(s)=\int_{0}^{s} \frac{d s^{\prime}}{\beta_{x}\left(s^{\prime}\right)}, \quad \mu_{y}(s)=\int_{0}^{s} \frac{d s^{\prime}}{\beta_{y}\left(s^{\prime}\right)}, \quad K(s)=\frac{\partial^{(l-1)} B}{\partial x^{(l-1)}},
\end{gathered}
$$

and $B=B_{y}\left(B_{x}\right)$ if $n$ is even (odd).

\subsection{Resonance Correction}

For the correction of the $m Q_{x}+n Q_{y}=N$ resonance we require

$$
\kappa_{0}+\kappa=0
$$

where $\kappa_{0}$ is the excitation coefficient due to imperfections in the machine, and $\kappa$ is produced by the resonance correction scheme. For a set of point correction elements we have

$$
\kappa=C \frac{K}{p} \sum_{j} I_{j} \beta_{x j}^{|m / 2|} \beta_{y j}^{|n / 2|} e^{i \psi_{j}},
$$

where $K$ is the integrated strength per unit current of each correction element, $I_{j}$ is the current in the $j$ th element, and

$$
\beta_{x j}=\beta_{x}\left(s_{j}\right), \quad \beta_{y j}=\beta_{y}\left(s_{j}\right), \quad \psi_{j}=\psi\left(s_{j}\right) .
$$

If the correction elements occupy the same relative positions in each of $P$ superperiods, and we let $q$ and $p$ denote the $q$ th correction element in the $p$ th superperiod, then (27) becomes

$$
\kappa=C \frac{K}{p} \sum_{p=1}^{P} \sum_{q} I_{p q} \beta_{x q}^{|m / 2|} \beta_{y q}^{|n / 2|} e^{i \psi_{p q}},
$$

where

$$
\psi_{p q}=\psi_{q}+2 \pi N(p-1) / P
$$

Now, if we choose

$$
I_{p q}=I_{q} \cos \frac{2 \pi M}{P}(p-1)=I_{q} f_{p}
$$


as in the correction scheme proposed in Ref. (2), then (29) becomes

$$
\kappa=C \frac{K}{p} S_{M N} \sum_{q} I_{q} \beta_{x q}^{|m / 2|} \beta_{y q}^{|n / 2|} e^{i \psi_{q}}
$$

where

$$
\begin{gathered}
S_{M N}=\sum_{p=1}^{P} e^{i 2 \pi N(p-1) / P} \cos \frac{2 \pi M}{P}(p-1)=\frac{1}{2} \sum_{p=1}^{P}\left(X^{p-1}+Y^{p-1}\right) \\
X=e^{i 2 \pi(N+M) / P}, \quad Y=e^{i 2 \pi(N-M) / P} .
\end{gathered}
$$

Here we see that since

$$
(Z-1) \sum_{p=1}^{P} Z^{p-1}=Z^{P}-1,
$$

$S_{M N}$ is zero unless $N \pm M=P I$, where $I$ is any integer. Thus $S_{M N}$ serves as a filter for unwanted harmonics. For the case of the Booster, $P=6$, and we find the following values for $S_{M N}$ and $f_{p}$ :

\begin{tabular}{|cc|c|cc|}
\hline $\mathrm{M}$ & $\mathrm{N}$ & $S_{M N}$ & $U_{M N}$ & $V_{M N}$ \\
\hline 14 & 14 & 3 & $3 / 2$ & $3 / 2$ \\
13 & 13 & 3 & $3 / 2$ & $3 / 2$ \\
13 & 5 & 3 & $3 / 2$ & $3 / 2$ \\
14 & 4 & 3 & $3 / 2$ & $3 / 2$ \\
9 & 9 & 6 & 3 & 3 \\
0 & 0 & 6 & 3 & 3 \\
\hline 13 & 14 & 0 & $3 / 2$ & $-3 / 2$ \\
14 & 13 & 0 & $3 / 2$ & $-3 / 2$ \\
14 & 5 & 0 & $3 / 2$ & $-3 / 2$ \\
13 & 4 & 0 & $3 / 2$ & $-3 / 2$ \\
9 & 0 & 0 & 3 & -3 \\
0 & 9 & 0 & 3 & -3 \\
\hline
\end{tabular}

and

\begin{tabular}{|c|cccccc|}
\hline $\mathrm{M}$ & $f_{1}$ & $f_{2}$ & $f_{3}$ & $f_{4}$ & $f_{5}$ & $f_{6}$ \\
\hline 14 & 1 & $-1 / 2$ & $-1 / 2$ & 1 & $-1 / 2$ & $-1 / 2$ \\
13 & 1 & $1 / 2$ & $-1 / 2$ & -1 & $-1 / 2$ & $1 / 2$ \\
9 & 1 & -1 & 1 & -1 & 1 & -1 \\
0 & 1 & 1 & 1 & 1 & 1 & 1 \\
\hline
\end{tabular}


where

$$
S_{M N}=U_{M N}+V_{M N}, \quad U_{M N}=\sum_{p=1}^{P / 2} e^{i 2 \pi N(2 p-2) / P} \cos \frac{2 \pi M}{P}(2 p-2) .
$$

\subsection{Correction of Resonances $3 Q_{x}=14, Q_{x}+2 Q_{y}=14$, $3 Q_{x}=13$, and $Q_{x}+2 Q_{y}=13$.}

These resonances are corrected with the eight strings of sextupoles listed in section 2.2. Since the auxiliary windings on the sextupoles in superperiods B, C, E, F have half as many turns as those in superperiods A and D, and are wired with the opposite polarity, $f_{p}$ will have the values tabulated for the case $M=14$ or $M=13$. For the correction of resonances $3 Q_{x}=14$ and $Q_{x}+2 Q_{y}=14$, the currents in each superperiod are given by (31) with $M=14$ and $I_{3}=-I_{1}, I_{4}=-I_{2}, I_{7}=-I_{5}, I_{8}=-I_{6}$. If $J_{1}, J_{2}, J_{3}, J_{4}$ are the currents in sextupoles $1,2,5,6$ of superperiod $A$, and $J_{5}, J_{6}, J_{7}$, $J_{8}$, are the currents in sextupoles $1,2,5,6$ of superperiod $D$, then (32) becomes

$$
\left(\begin{array}{l}
C X_{14} \\
S X_{14} \\
C Y_{14} \\
S Y_{14}
\end{array}\right)=\frac{C}{2}\left(\frac{S}{p}\right) \mathbf{M}_{14}\left(\begin{array}{l}
J_{1}+J_{5} \\
J_{2}+J_{6} \\
J_{3}+J_{7} \\
J_{4}+J_{8}
\end{array}\right),
$$

where $C X_{14}$ and $S X_{14}$ are the real and imaginary parts of the excitation coefficient for the $3 Q_{x}=14$ resonance, $C Y_{14}$ and $S Y_{14}$ are the real and imaginary parts of $-1 / 3$ times the excitation coefficient for the $Q_{x}+2 Q_{y}=14$ resonance, and

$$
\begin{gathered}
\mathbf{M}_{14}=3\left(\mathbf{M}_{a}-\mathbf{M}_{b}\right), \\
\mathbf{M}_{a}=\left(\begin{array}{cccc}
\beta_{x 1}^{3 / 2} C_{x 1} & \beta_{x 2}^{3 / 2} C_{x 2} & \beta_{x 5}^{3 / 2} C_{x 5} & \beta_{x 6}^{3 / 2} C_{x 6} \\
\beta_{x 1}^{3 / 2} S_{x 1} & \beta_{x 2}^{3 / 2} S_{x 2} & \beta_{x 5}^{3 / 2} S_{x 5} & \beta_{x 6}^{3 / 2} S_{x 6} \\
\beta_{x 1}^{1 / 2} \beta_{y 1} C_{y 1} & \beta_{x 2}^{1 / 2} \beta_{y 2} C_{y 2} & \beta_{x 5}^{1 / 2} \beta_{y 5} C_{y 5} & \beta_{x 6}^{1 / 2} \beta_{y 6} C_{y 6} \\
\beta_{x 1}^{1 / 2} \beta_{y 1} S_{y 1} & \beta_{x 2}^{1 / 2} \beta_{y 2} S_{y 2} & \beta_{x 5}^{1 / 2} \beta_{y 5} S_{y 5} & \beta_{x 6}^{1 / 2} \beta_{y 6} S_{y 6}
\end{array}\right), \\
\mathbf{M}_{b}=\left(\begin{array}{cccc}
\beta_{x 3}^{3 / 2} C_{x 3} & \beta_{x 4}^{3 / 2} C_{x 4} & \beta_{x 7}^{3 / 2} C_{x 7} & \beta_{x 8}^{3 / 2} C_{x 8} \\
\beta_{x 3}^{3 / 2} S_{x 3} & \beta_{x 4}^{3 / 2} S_{x 4} & \beta_{x 7}^{3 / 2} S_{x 7} & \beta_{x 8}^{3 / 2} S_{x 8} \\
\beta_{x 3}^{1 / 2} \beta_{y 3} C_{y 3} & \beta_{x 4}^{1 / 2} \beta_{y 4} C_{y 4} & \beta_{x 7}^{1 / 2} \beta_{y 7} C_{y 7} & \beta_{x 8}^{1 / 2} \beta_{y 8} C_{y 8} \\
\beta_{x 3}^{1 / 2} \beta_{y 3} S_{y 3} & \beta_{x 4}^{1 / 2} \beta_{y 4} S_{y 4} & \beta_{x 7}^{1 / 2} \beta_{y 7} S_{y 7} & \beta_{x 8}^{1 / 2} \beta_{y 8} S_{y 8}
\end{array}\right),
\end{gathered}
$$




$$
\begin{gathered}
C_{x j}=\cos \psi_{x j}, \quad S_{x j}=\sin \psi_{x j}, \quad C_{y j}=\cos \psi_{y j}, \quad S_{y j}=\sin \psi_{y j}, \\
\psi_{x j}=3 \mu_{x j}+\left(N-3 Q_{x}\right) \theta_{j}, \quad \psi_{y j}=\mu_{x j}+2 \mu_{y j}+\left(N-Q_{x}-2 Q_{y}\right) \theta_{j} .
\end{gathered}
$$

Similarly, for the correction of the $3 Q_{x}=13$ and $Q_{x}+2 Q_{y}=13$

resonances we have

$$
\left(\begin{array}{c}
C X_{13} \\
S X_{13} \\
C Y_{13} \\
S Y_{13}
\end{array}\right)=\frac{C}{2}\left(\frac{S}{p}\right) \mathbf{M}_{13}\left(\begin{array}{c}
J_{1}-J_{5} \\
J_{2}-J_{6} \\
J_{3}-J_{7} \\
J_{4}-J_{8}
\end{array}\right),
$$

where $\mathrm{M}_{13}$ is given by (38-42) with $N=13$.

Now, since $S_{M N}=3$ for $(M, N)=(14,4)$ and $(13,5)$, correction of the $3 Q_{x}=14$ and $Q_{x}+2 Q_{y}=14$ resonances can produce a nonzero excitation coefficient for the $2 Q_{y}-Q_{x}=4$ resonance, and correction of the $3 Q_{x}=13$ and $Q_{x}+2 Q_{y}=13$ resonances can produce a nonzero excitation coefficient for the $2 Q_{y}-Q_{x}=5$ resonance. Evaluating (32) for the $2 Q_{y}-Q_{x}=4$ resonance we find

$$
\left(\begin{array}{c}
C X Y_{4} \\
S X Y_{4}
\end{array}\right)=\frac{C}{2}\left(\frac{S}{p}\right) \mathbf{M}_{4}\left(\begin{array}{c}
J_{1}+J_{5} \\
J_{2}+J_{6} \\
J_{3}+J_{7} \\
J_{4}+J_{8}
\end{array}\right)
$$

where $C X Y_{4}$ and $S X Y_{4}$ are the real and imaginary parts of $-1 / 3$ times the excitation coefficient for the $2 Q_{y}-Q_{x}=N=4$ resonance, and

$$
\begin{gathered}
\mathbf{M}_{4}=3\left(\mathbf{M}_{a}-\mathbf{M}_{b}\right), \\
\mathbf{M}_{a}=\left(\begin{array}{cccc}
\beta_{x 1}^{1 / 2} \beta_{y 1} C_{1} & \beta_{x 2}^{1 / 2} \beta_{y 2} C_{2} & \beta_{x 5}^{1 / 2} \beta_{y 5} C_{5} & \beta_{x 6}^{1 / 2} \beta_{y 6} C_{6} \\
\beta_{x 1}^{1 / 2} \beta_{y 1} S_{1} & \beta_{x 2}^{1 / 2} \beta_{y 2} S_{2} & \beta_{x 5}^{1 / 2} \beta_{y 5} S_{5} & \beta_{x 6}^{1 / 2} \beta_{y 6} S_{6}
\end{array}\right), \\
\mathbf{M}_{b}=\left(\begin{array}{cccc}
\beta_{x 3}^{1 / 2} \beta_{y 3} C_{3} & \beta_{x 4}^{1 / 2} \beta_{y 4} C_{4} & \beta_{x 7}^{1 / 2} \beta_{y 7} C_{7} & \beta_{x 8}^{1 / 2} \beta_{y 8} C_{8} \\
\beta_{x 3}^{1 / 2} \beta_{y 3} S_{3} & \beta_{x 4}^{1 / 2} \beta_{y 4} S_{4} & \beta_{x 7}^{1 / 2} \beta_{y 7} S_{7} & \beta_{x 8}^{1 / 2} \beta_{y 8} S_{8}
\end{array}\right), \\
C_{j}=\cos \psi_{j}, \quad S_{j}=\sin \psi_{j}, \quad \psi_{j}=2 \mu_{y j}-\mu_{x j}+\left(N-2 Q_{y}+Q_{x}\right) \theta_{j} .
\end{gathered}
$$

Similarly, for the $2 Q_{y}-Q_{x}=5$ resonance we have

$$
\left(\begin{array}{c}
C X Y_{5} \\
S X Y_{5}
\end{array}\right)=\frac{C}{2}\left(\frac{S}{p}\right) \mathbf{M}_{5}\left(\begin{array}{l}
J_{1}-J_{5} \\
J_{2}-J_{6} \\
J_{3}-J_{7} \\
J_{4}-J_{8}
\end{array}\right),
$$

where $\mathrm{M}_{5}$ is given by (45-48) with $N=5$. 


\subsection{Correction of Resonances $Q_{x}-Q_{y}=0$ and $Q_{x}+Q_{y}=9$.}

These resonances are corrected with the four strings of skew quadrupoles listed in section 3.2. (Only the skew quads in sections $1,2,7,8$ of each superperiod are used.) These strings can be excited so that $f_{p}$ will have the values tabulated for the case $M=0$ or $M=9$. The currents in each superperiod are given by (31) with $M=0$ or $M=9$ and $I_{1}=I_{7}, I_{2}=I_{8}$. If $J_{1}$ and $J_{2}$ are the currents in skew quads 1 and 2 of superperiod $A$, and $J_{3}$ and $J_{4}$ are the currents in skew quads 1 and 2 of superperiod $\mathrm{B}$, then (32) becomes

$$
\left(\begin{array}{c}
C_{0} \\
S_{0}
\end{array}\right)=\frac{C}{2}\left(\frac{Q}{p}\right) \mathbf{N}_{1}\left(\begin{array}{c}
J_{1}+J_{3} \\
J_{2}+J_{4}
\end{array}\right)
$$

and

$$
\left(\begin{array}{c}
C_{9} \\
S_{9}
\end{array}\right)=\frac{C}{2}\left(\frac{Q}{p}\right) \mathbf{N}_{2}\left(\begin{array}{c}
J_{1}-J_{3} \\
J_{2}-J_{4}
\end{array}\right)
$$

where $C_{0}$ and $S_{0}$ are the real and imaginary parts of the excitation coefficient for the $Q_{x}-Q_{y}=0$ resonance, $C_{9}$ and $S_{9}$ are the real and imaginary parts of the excitation coefficient for the $Q_{x}+Q_{y}=9$ resonance, and

$$
\begin{aligned}
\mathbf{N}_{1} & =6\left(\begin{array}{cc}
\beta_{x 1}^{1 / 2} \beta_{y 1}^{1 / 2} C_{11}+\beta_{x 7}^{1 / 2} \beta_{y 7}^{1 / 2} C_{17} & \beta_{x 2}^{1 / 2} \beta_{y 2}^{1 / 2} C_{12}+\beta_{x 8}^{1 / 2} \beta_{y 8}^{1 / 2} C_{18} \\
\beta_{x 1}^{1 / 2} \beta_{y 1}^{1 / 2} S_{11}+\beta_{x 7}^{1 / 2} \beta_{y 7}^{1 / 2} S_{17} & \beta_{x 2}^{1 / 2} \beta_{y 2}^{1 / 2} S_{12}+\beta_{x 8}^{1 / 2} \beta_{y 8}^{1 / 2} S_{18}
\end{array}\right) \\
\mathbf{N}_{2} & =6\left(\begin{array}{cc}
\beta_{x 1}^{1 / 2} \beta_{y 1}^{1 / 2} C_{21}+\beta_{x 7}^{1 / 2} \beta_{y 7}^{1 / 2} C_{27} & \beta_{x 2}^{1 / 2} \beta_{y 2}^{1 / 2} C_{22}+\beta_{x 8}^{1 / 2} \beta_{y 8}^{1 / 2} C_{28} \\
\beta_{x 1}^{1 / 2} \beta_{y 1}^{1 / 2} S_{21}+\beta_{x 7}^{1 / 2} \beta_{y 7}^{1 / 2} S_{27} & \beta_{x 2}^{1 / 2} \beta_{y 2}^{1 / 2} S_{22}+\beta_{x 8}^{1 / 2} \beta_{y 8}^{1 / 2} S_{28}
\end{array}\right) \\
C_{n j}=\cos \psi_{n j}, & S_{n j}=\sin \psi_{n j}
\end{aligned}
$$

\subsection{Correction of Resonances $2 Q_{x}=9$ and $2 Q_{y}=9$.}

The $2 Q_{x}=9$ and $2 Q_{y}=9$ resonances are corrected with the four strings of quadrupoles listed in section 1.2. Since these strings are wired so that the auxiliary windings on the quads in superperiods $\mathrm{B}, \mathrm{D}, \mathrm{F}$ have polarity opposite those in superperiods $\mathrm{A}, \mathrm{C}, \mathrm{E}, f_{p}$ will have the values tabulated for the case $M=9$. The currents in each superperiod are given by (31) 
with $M=9$ and $I_{1}=I_{7}, I_{2}=I_{8}, I_{3}=-I_{5}, I_{4}=-I_{6}$. If $J_{1}, J_{2}, J_{3}, J_{4}$ are the currents in quadrupoles $1,2,3,4$ of superperiod $A$, then (32) becomes

$$
\left(\begin{array}{c}
C X \\
S X \\
C Y \\
S Y
\end{array}\right)=\left(\frac{C}{p}\right) \mathbf{M Q}\left(\begin{array}{c}
J_{1} \\
J_{2} \\
J_{3} \\
J_{4}
\end{array}\right)
$$

where $\mathbf{Q}$ is given by (3), $C X$ and $S X$ are the real and imaginary parts of the excitation coefficient for the $2 Q_{x}=9$ resonance, $C Y$ and $S Y$ are the real and imaginary parts of the excitation coefficient for the $2 Q_{y}=9$ resonance, and

$$
\begin{aligned}
& M=6\left(M_{a}+M_{b}\right), \\
& \mathbf{M}_{u}=\left(\begin{array}{cccc}
\beta_{x 1} C_{x 1} & \beta_{x 2} C_{x 2} & \beta_{x 3} C_{x 3} & \beta_{x 4} C_{x 4} \\
\beta_{x 1} S_{x 1} & \beta_{x 2} S_{x 2} & \beta_{x 3} S_{x 3} & \beta_{x 4} S_{x 4} \\
\beta_{y 1} C_{y 1} & \beta_{y 2} C_{y 2} & \beta_{y 3} C_{y 3} & \beta_{y 4} C_{y 4} \\
\beta_{y 1} S_{y 1} & \beta_{y 2} S_{y 2} & \beta_{y 3} S_{y 3} & \beta_{y 4} S_{y 4}
\end{array}\right), \\
& \mathbf{M}_{b}=\left(\begin{array}{cccc}
\beta_{x 7} C_{x 7} & \beta_{x 8} C_{x 8} & -\beta_{x 5} C_{x 5} & -\beta_{x 6} C_{x 6} \\
\beta_{x 7} S_{x 7} & \beta_{x 8} S_{x 8} & -\beta_{x 5} S_{x 5} & -\beta_{x 6} S_{x 6} \\
\beta_{y 7} C_{y 7} & \beta_{y 8} C_{y 8} & -\beta_{y 5} C_{y 5} & -\beta_{y 6} C_{y 6} \\
\beta_{y 7} S_{y 7} & \beta_{y 8} S_{y 8} & -\beta_{y 5} S_{y 5} & -\beta_{y 6} S_{y 6}
\end{array}\right), \\
& C_{x j}=\cos \psi_{x j}, \quad S_{x j}=\sin \psi_{x j}, \quad C_{y j}=\cos \psi_{y j}, \quad S_{y j}=\sin \psi_{y j}, \\
& \psi_{x j}=2 \mu_{x j}+\left(N-2 Q_{x}\right) \theta_{j}, \quad \psi_{y j}=2 \mu_{y j}+\left(N-2 Q_{y}\right) \theta_{j}, \quad(N=9)
\end{aligned}
$$

\section{References}

1. C. J. Gardner, 'Effective Placement of Stopband Correction Elements in an AGS Lattice', AGS/AD/Tech. Note No. 321, 30 May 1989.

2. S. Tepikian, 'An Upgraded Version of the Software for Resonance Correction', unpublished note.

3. S. Tepikian, unpublished resonance strength calculations.

4. E. Bleser, 'Booster Short Quadrupole Production Measurements', Booster Tech. Note No. 174, 12 September 1990.

5. E. Bleser, 'Booster Long Quadrupole Production Measurements', Booster Tech. Note No. 176, 13 September 1990. 
6. F. Bleser, 'Booster Sextupole Production Measurements', Booster Tech. Note No. 182, 13 March 1992.

7. E. Bleser, 'Corrector Package Production Measurements', Booster Tech. Note No.

8. G. Guignard, 'The General Theory of all Sum and Difference Resonances in a Three-Dimensional Magnetic Field in a Synchrotron', CERN 76-06, 23 March 1976; 'A General Treatment of Resonances in Accelerators' CERN 78-11, 10 November 1978.

9. W.T. Weng, 'Space Charge Effects-Tune Shifts and Resonances', Physics of Particle Accelerators, AIP Conference Proceedings 153, American Institute of Physics, New York, 1987, pp. 348-389. 